\title{
On the Dynamical 4D BTZ Black Hole Solution in Conformally Invariant Gravity
}

\author{
Reinoud J. Slagter ${ }^{1,2}$ \\ ${ }^{1}$ Asfyon, Astronomisch Fysisch Onderzoek Nederland, Bussum, The Netherlands \\ ${ }^{2}$ University of Amsterdam, Amsterdam, The Netherlands \\ Email:info@asfyon.com
}

How to cite this paper: Slagter, R.J. (2020) On the Dynamical 4D BTZ Black Hole Solution in Conformally Invariant Gravity. Journal of Modern Physics, 11, 1711-1730. https://doi.org/10.4236/jmp.2020.1110105

Received: September 11, 2020

Accepted: October 23, 2020

Published: October 26, 2020

Copyright $\odot 2020$ by author(s) and Scientific Research Publishing Inc. This work is licensed under the Creative Commons Attribution International License (CC BY 4.0).

http://creativecommons.org/licenses/by/4.0/

(c) (i) Open Access

\begin{abstract}
We review the $(2+1)$-dimensional Baňados-Teitelboim-Zanelli black hole solution in conformally invariant gravity, uplifted to $(3+1)$-dimensional spacetime. For the matter content we use a scalar-gauge field. The metric is written as $g_{\mu \nu}=\omega^{2} \tilde{g}_{\mu v}$, where the dilaton field $\omega$ contains all the scale dependencies and where $\tilde{g}_{\mu v}$ represents the "un-physical" spacetime. A numerical solution is presented and shows how the dilaton can be treated on equal footing with the scalar field. The location of the apparent horizon and ergo-surface depends critically on the parameters and initial values of the model. It is not a hard task to find suitable initial parameters in order to obtain a regular and singular free $g_{\mu \nu}$ out of a BTZ-type solution for $\tilde{g}_{\mu \nu}$. In the vacuum situation, an exact time-dependent solution in the Eddington-Finkelstein coordinates is found, which is valid for the $(2+1)$-dimensional BTZ spacetime as well as for the uplifted $(3+1)$-dimensional BTZ spacetime. While $\tilde{g}_{\mu v}$ resembles the standard BTZ solution with its horizons, $g_{\mu v}$ is flat. The dilaton field becomes an infinitesimal renormalizable quantum field, which switches on and off Hawking radiation. This solution can be used to investigate the small distance scale of the model and the black hole complementarity issues. It can also be used to describe the problem of how to map the quantum states of the outgoing radiation as seen by a distant observer and the ingoing by a local observer in a one-to-one way. The two observers will use a different conformal gauge. A possible connection is made with the antipodal identification and unitarity issues. This research shows the power of conformally invariant gravity and can be applied to bridge the gap between general relativity and quantum field theory in the vicinity of the horizons of black holes.
\end{abstract}

\section{Keywords}

Scalar-Gauge Field, Baňados-Teitelboim-Zanelli Black Hole, Conformal 
Invariance, Dilaton Field, Eddington-Finkelstein Coordinate, Black Hole Complementarity, Antipodal Identification

\section{Introduction}

Besides the well-studied Schwarzschild and Kerr solution in general relativity theory (GRT), there is another black hole solution in $(2+1)$-dimensional spacetime, i.e., the Baňados-Teitelboim-Zanelli (BTZ) black hole [1] [2]. The BTZ geometry solves Einstein's equations with a negative cosmological constant in $(2+1)$-dimensions. In general, $(2+1)$-dimensional gravity has been widely recognized as a laboratory not only for studying GRT, but also quantum-gravity models. A nice overview of these models can be found in the book of Compère [3]. It is conjectured that this genuine solution will be of importance when one considers thermodynamic properties close to the horizon, i.e., Hawking radiation. The $(2+1)$-dimensional BTZ solution is comparable with the spinning point particle solution (or "cosmon" [4]) of the dimensional reduced spinning cosmic string or Kerr solution. $(2+1)$-dimensional gravity without matter, implying that the Ricci- and Riemann tensor vanish, so matter-free regions are flat pieces of spacetime. When locally a mass at rest is present, it cuts out a wedge from the 2-dimensional space surrounding it and makes the space conical. The angle deficit is then proportional to the mass [5]. The important fact is that the spinning point particle has a physical acceptable counterpart in $(3+1)$-dimensions, i.e., the spinning cosmic string. The z-coordinate is suppressed, because there is no structure in that direction altogether. It is not a surprise that these models are used in constructing quantum gravity models. In these models one uses locally Minkowski spacetime, so planar gravity fits in very well.

The BTZ solution is related to the Anti-deSitter/Conformal Field Theory (AdS/CFT) correspondence [6] and became a tool to understand black hole entropy [7]. For the $(2+1)$-dimensional BTZ black hole solution, one can try to follow the same procedure as used for the cosmic string, by uplifting the solution to $(3+1)$-dimensional spacetime. However, the cosmological constant must be taken zero, when the BTZ solution is uplifted, so it loses its connection with the asymptotic $\mathrm{AdS}_{3}$ black hole. This opens the way to new solutions, which was done in a conformally invariant setting [8] [9]. Conformal invariance (CI) was originally introduced by Weyl [10]. See also the textbook of Wald [11]. The AdS/CFT correspondence renewed the interest in conformal gravity. AdS/CFT is a conjectured relationship between two kinds of physical theories. AdS spaces are used in theories of quantum gravity while CFT includes theories similar to the Yang Mills theories that describe elementary particles. It is believed that CI can help us to move a little further along the road to quantum gravity. Exact local CI at the level of the Lagrangian, will then spontaneously be broken, comparable with the Brout-Englert-Higgs (BEH) mechanism. It is an approved alterna- 
tive for disclosing the small-distance structure when one tries to describe quantum-gravity problems [12] [13]. It can also be used to model scale-invariance in the cosmic microwave background radiation (CMBR) [14]. Another interesting application can be found in the work of Mannheim on conformal cosmology [15]. This model could serve as an alternative approach to explain the rotational curves of galaxies, without recourse to dark matter and dark energy (or cosmological constant). In Mannheim's model, standard Schwarzschild phenomenology can in fact be recovered in conformal gravity in the presence of a scalar-gauge field. Further, GRT will be different on different scales by virtue of the dilaton field. As regards dark matter, there is nothing in principle wrong with the existence of nonluminous material. Rather, what is disturbing is the ad hoc, after the fact, way in which dark matter is actually introduced, with its presence only being inferred after known luminous astrophysical sources are found to fail to account for any given astrophysical observation [15]. The dark energy problem is even more severe, and not simply because its composition and nature are as mysterious as that of dark matter. The introduction of a cosmological constant will not solve this problem. It is not possible to explain the huge discrepancy between the contribution from zero-point fluctuations in quantum field theory and the predicted value in GRT (some 120 orders of magnitude!). It is hoped that in future more data will become available for the rotation curves of galaxies. The validity of conformal gravity theory can also be tested with the cosmic microwave background. It is a challenge to alternate theories to fit the cosmic microwave background data. The growth of inhomogeneities in the model and the size of the fluctuation "yardstick" (determined by $\omega$ ) of the conformal theory would be different from the one used in the standard theory.

Another key problem is the handling of asymptotic flatness of isolated systems in GRT, especially when they radiate and the generation of the metric $g_{\mu v}$ from at least Ricci-flat spacetime. In the non-vacuum case one should construct a Lagrangian where spacetime and the fields defined on it, are topological regular and physical acceptable. This can be done by considering the scale factor (or warp factor in higher-dimensional models [16]) as a dilaton field besides, for example, a conformally coupled scalar field or other fields. Conformal invariant gravity distinguishes itself by the notion that the spacetime is written as $g_{\mu v}=\omega^{2} \tilde{g}_{\mu v}$, with $\omega$ a dilaton field which contains all the scale dependencies and $\tilde{g}_{\mu v}$ the "un-physical' spacetime, related to the $(2+1)$-dimensional Kerr and BTZ black hole solution. $\omega$ is just an ordinary renormalizable field, which could create the spacetime twofold: an in-falling and outside observer use different ways to fix the conformal gauge in order to overcome the unitarity problems encountered in standard approaches in quantum gravity models. It can be handled on equal footing with a scalar field. Renormalization and unitarity problems in general relativity at the quantum scale, have a long history [17] [18]. In first instance, it was believed that conformal invariance would not survive in quantum gravity (see, for example, the overview of Duff [19]). However, new 
interest occurred, when it was realized that Weyl anomalies and unitarity problems could be overcome. In constructing an effective theory in canonical quantum gravity and to obtain quantum amplitudes, one performs a functional integration of the exponent of the entire action over, for example, all components of the metric tensor at all spacetime points. Now the integration is first performed over the dilaton function $\omega$ together with the matter fields. Integration over the $\omega$ is identical to the integration over a renormalizable scalar field. In the action the dilaton must be shifted to the complex contour, in order to obtain the same unitarity and positivity features as the scalar field. Another actual problem is the back hole complementarity: how to handle the in- and out-going radiation as experienced by an in-falling- and outside observer. In a dynamical setting, there will be a back-reaction on the location of the horizon(s). The in falling and outside observer will experience a different $\omega$. They use different ways to fix the conformal gauge. Further, there is the problem of extending the Penrose diagram in a one-to-one map, in order to avoid unitarity and locality problems and to avoid the need to define the inside of the black hole (or even another universe). The antipodal identification could be used [20], i.e., a conformal compactification of the manifold [21].

In Section 2 and 3 we describe the dynamical CI model on the original BTZ black hole spacetime, uplifted to $(3+1)$ dimensions. In Section 3.2 we present a numerical solution of the complete set of coupled PDE's. In Section 4 and 5 we find an exact time-dependent solution in the vacuum situation in Eddington-Finkelstein coordinates and we explain possible ways to connect this solution with recent research on black hole complementarity, antipodal identifications and hawking radiation.

\section{The BTZ Solution Revised}

If one solves the Einstein equations $G_{\mu \nu}=\lambda g_{\mu \nu}$ for the spacetime

$$
\mathrm{d} s^{2}=-N(\rho)^{2} \mathrm{~d} t^{2}+\frac{1}{N(\rho)^{2}} \mathrm{~d} \rho^{2}+\rho^{2}\left(\mathrm{~d} \varphi+N^{\varphi}(\rho) \mathrm{d} t\right)^{2},
$$

one obtains [8]

$$
\begin{aligned}
& N(\rho)^{2} \equiv \alpha^{2}-\Lambda \rho^{2}+\frac{16 G^{2} J^{2}}{\rho^{2}}, \\
& N^{\varphi}(\rho) \equiv-\frac{4 G J}{\rho^{2}}+S,
\end{aligned}
$$

where $S, J$ and $\alpha$ are integration constants [1] [3]. The parameters $\alpha$ and $J$ represent the standard ADM mass $\left(\alpha^{2}= \pm 8 G M\right)$ and angular momentum and determine the asymptotic behavior of the solution. $\Lambda$ represents the cosmological constant. There is an inner and outer horizon and an ergo-circle just as in the case of the Kerr spacetime. However, we live in a 4-dimensional spacetime, so one way or another, the BTZ solution in 3 dimensions must be up-lifted to $(3+1)$ dimensions. From the Einstein equations one can then easily verify that $\Lambda=0$. So we consider here the case $\Lambda=0$, and we write the spacetime as 


$$
\begin{aligned}
\mathrm{d} s^{2}= & -\left[8 G(J S-M)-S^{2} \rho^{2}\right] \mathrm{d} t^{2}+\frac{\rho^{2} r_{H}^{2}}{16 G^{2} J^{2}\left(\rho_{H}^{2}-r^{2}\right)} \mathrm{d} \rho^{2} \\
& +\rho^{2} \mathrm{~d} \varphi^{2}+2 \rho^{2}\left(S-\frac{4 G J}{\rho^{2}}\right) \mathrm{d} t \mathrm{~d} \varphi,
\end{aligned}
$$

with $\rho_{H}$ the horizon $\rho_{H}=\sqrt{\frac{2 G}{M}} J$. In the case of $S=0$, which is also done in the original BTZ solution, one can transform the spacetime to

$$
\mathrm{d} s^{2}=-\left(\alpha \mathrm{d} t+\frac{4 G J}{\alpha} \mathrm{d} \varphi\right)^{2}+\mathrm{d}\left(\rho^{\prime}\right)^{2}+\alpha^{2}\left(\rho^{\prime}\right)^{2} \mathrm{~d} \varphi^{2},
$$

by $\left(\rho^{\prime}\right)^{2}=\frac{16 G^{2} J^{2}+\alpha^{2} \rho^{2}}{\alpha^{4}}$. This is just the spinning particle spacetime [4].

In a former study [8], we investigated the revised BTZ solution in connection with the spinning cosmic strings and conformal invariance and found an uplifted exact vacuum solution.

The spacetime Equation (1) is then replaced by

$$
\mathrm{d} s^{2}=\omega(\rho)^{2}\left[-N(\rho)^{2} \mathrm{~d} t^{2}+\frac{1}{N(\rho)^{2}} \mathrm{~d} \rho^{2}+\rho^{2}\left(\mathrm{~d} \varphi+N^{\varphi}(\rho) \mathrm{d} t\right)^{2}\right],
$$

with $\omega$ the dilaton field. A typical solution is then found [8] for $\omega, N$ and $N^{\varphi}$, which is asymptotically regular. See Figure 1 . It is remarkable that this solution resembles the standard BTZ solution. However, we don't need a cosmological constant. Further, $\omega$ plays the role here of a cosmological scale factor. So we can extend the model to small scales. This will be done, in a dynamical setting, in the next section.

\section{The Dynamical BTZ Model}

\subsection{The Field Equations}

Let us consider the time-dependent spacetime $g_{\mu v} \equiv \omega(t, \rho)^{2} \tilde{g}_{\mu v}$

$$
\mathrm{d} s^{2}=\omega(t, \rho)^{2}\left[-N(t, \rho)^{2} \mathrm{~d} t^{2}+\frac{1}{N(t, \rho)^{2}} \mathrm{~d} \rho^{2}+\mathrm{d} z^{2}+\rho^{2}\left(\mathrm{~d} \varphi+N^{\varphi}(t, \rho) \mathrm{d} t\right)^{2}\right],
$$
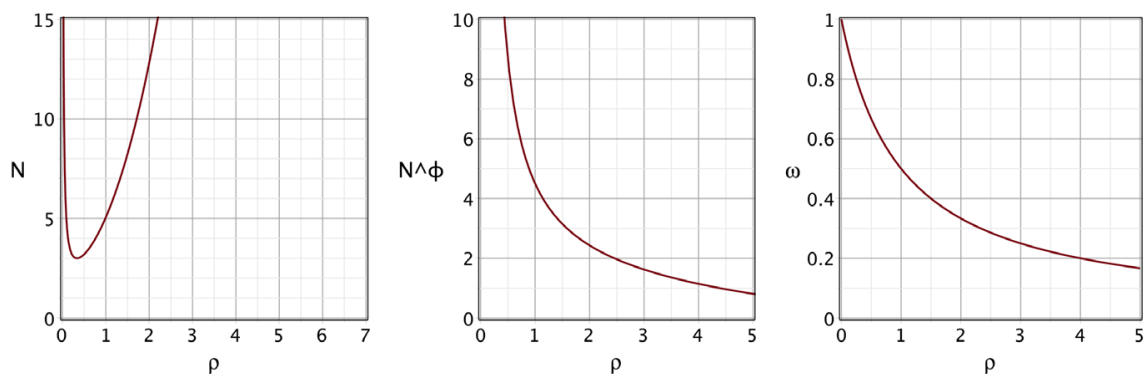

Figure 1. Example of a conformal invariant solution of the BTZ on a 3-dimensional spacetime. $N \sim \frac{(r+c)^{4}}{r}$. The location of the horizon is determined by the constant $c$. 
with $\omega$ the dilaton field. The action under consideration is

$$
\begin{aligned}
S= & \int \mathrm{d}^{4} x \sqrt{-\tilde{g}}\left\{-\frac{1}{12}\left(\tilde{\Phi} \tilde{\Phi}^{*}+\bar{\omega}^{2}\right) \tilde{R}-\frac{1}{2}\left(D_{\alpha} \tilde{\Phi}\left(D^{\alpha} \tilde{\Phi}\right)^{*}+\partial_{\alpha} \bar{\omega} \partial^{\alpha} \bar{\omega}\right)\right. \\
& \left.-\frac{1}{4} F_{\alpha \beta} F^{\alpha \beta}-V(\tilde{\Phi}, \bar{\omega})-\frac{1}{36} \kappa^{2} \Lambda \bar{\omega}^{4}\right\} .
\end{aligned}
$$

We parameterize the scalar and gauge field as

$$
A_{\mu}=\left[P_{0}(t, \rho), 0,0, \frac{1}{e}(P(t, \rho)-n)\right], \quad \tilde{\Phi}(t, \rho)=\eta X(t, \rho) \mathrm{e}^{i n \varphi} .
$$

The gauge covariant derivative is $D_{\mu} \Phi=\tilde{\nabla}_{\mu} \Phi+i e A_{\mu} \Phi$ and $F_{\mu v}$ the Abelian field strength.

In the action one redefined $\bar{\omega}^{2} \equiv-\frac{6 \omega^{2}}{\kappa^{2}}$ (in order to ensure that the $\omega$ field has the same unitarity and positivity properties as the scalar field $\Phi[22])$ and $\Phi=\frac{1}{\omega} \tilde{\Phi}$. This Lagrangian is local conformal invariant under the transformation $\quad \tilde{g}_{\mu v} \rightarrow \Omega^{2} \tilde{g}_{\mu v}, \tilde{\Phi} \rightarrow \frac{1}{\Omega} \tilde{\Phi}$ and $\bar{\omega} \rightarrow \frac{1}{\Omega} \bar{\omega}$

Varying the Lagrangian with respect to $\tilde{g}_{\mu \nu}, \tilde{\Phi}, \bar{\omega}$ and $A_{\mu}$, we obtain the equations

$$
\begin{gathered}
\tilde{G}_{\mu \nu}=\frac{1}{\bar{\omega}^{2}+\tilde{\Phi} \tilde{\Phi}^{*}}\left(\tilde{T}_{\mu \nu}^{(\bar{\omega})}+\tilde{T}_{\mu \nu}^{(\tilde{\Phi}, c)}+\tilde{T}_{\mu \nu}^{(A)}+\frac{1}{6} \tilde{g}_{\mu \nu} \Lambda \kappa^{2} \bar{\omega}^{4}+\tilde{g}_{\mu \nu} V(\tilde{\Phi}, \bar{\omega})\right) \\
\tilde{\nabla}^{\alpha} \partial_{\alpha} \bar{\omega}-\frac{1}{6} \tilde{R} \bar{\omega}-\frac{\partial V}{\partial \bar{\omega}}-\frac{1}{9} \Lambda \kappa^{2} \bar{\omega}^{3}=0 \\
\tilde{D}^{\alpha} \tilde{D}_{\alpha} \tilde{\Phi}-\frac{1}{6} \tilde{R} \tilde{\Phi}-\frac{\partial V}{\partial \tilde{\Phi}^{*}}=0, \quad \tilde{\nabla}^{v} F_{\mu \nu}=\frac{i}{2} \varepsilon\left(\tilde{\Phi}\left(\tilde{D}_{\mu} \tilde{\Phi}\right)^{*}-\tilde{\Phi}^{*} \tilde{D} \tilde{\Phi}\right)
\end{gathered}
$$

with

$$
\begin{gathered}
\tilde{T}_{\mu \nu}^{(A)}=F_{\mu \alpha} F_{v}^{\alpha}-\frac{1}{4} \tilde{g}_{\mu \nu} F_{\alpha \beta} F^{\alpha \beta} \\
\tilde{T}_{\mu \nu}^{(\tilde{\Phi}, c)}=\left(\tilde{\nabla}_{\mu} \partial_{v} \tilde{\Phi} \tilde{\Phi}^{*}-\tilde{g}_{\mu \nu} \tilde{\nabla}_{\alpha} \partial^{\alpha} \tilde{\Phi} \tilde{\Phi}^{*}\right) \\
-3\left[\tilde{D}_{\mu} \tilde{\Phi}\left(\tilde{D}_{v} \tilde{\Phi}\right)^{*}+\left(\tilde{D}_{\mu} \tilde{\Phi}\right)^{*} \tilde{D}_{v} \tilde{\Phi}-\tilde{g}_{\mu \nu} \tilde{D}_{\alpha} \tilde{\Phi}\left(\tilde{D}^{\alpha} \tilde{\Phi}\right)^{*}\right]
\end{gathered}
$$

and

$$
\tilde{T}_{\mu \nu}^{(\bar{\omega})}=\left(\tilde{\nabla}_{\mu} \partial_{\nu} \bar{\omega}^{2}-\tilde{g}_{\mu \nu} \tilde{\nabla}_{\alpha} \partial^{\alpha} \bar{\omega}^{2}\right)-6\left(\partial_{\mu} \bar{\omega} \partial_{\nu} \bar{\omega}-\frac{1}{2} \tilde{g}_{\mu \nu} \partial_{\alpha} \bar{\omega} \partial^{\alpha} \bar{\omega}\right) .
$$

The covariant derivatives are taken with respect to $\tilde{g}_{\mu v}$. Newton's constant reappears in the quadratic interaction term for the scalar field. One refers to the field $\bar{\omega}(t, \rho)$ as a dilaton field. A massive term in $V(\tilde{\Phi}, \bar{\omega})$ will break the tracelessness of the energy momentum tensor, a necessity for conformal invariance. The cosmological constant $\Lambda$ could be ignored from the point of view of naturalness in order to avoid the inconceivable fine-tuning. Putting $\Lambda$ zero increases the symmetry of the model. 
Note that we cannot use in the stationary CI invariant model the gauge $A_{t}=0$. In standard gauged vortices models, this gauge simplifies the well-known Nielsen-Olesen $n=1$ vortex solution. The spatial rotational symmetry can then be completely compensated by a spatially uniform gauge transformation. In the stationary situation this is not the case.

For the Maxwell field $A_{\mu}$, the equation for $A_{t}$ is a constraint equation and in a time-dependent setting, only $A_{i}$ are dynamical. Standard, one uses then the Lorentz-gauge to remove $A_{t}$ completely. Gauge invariance is necessary in order to overcome breaking of locality and unitarity. In models with arbitrary vorticity $\mathrm{n}$ and SU(2)-Yang-Mills-Higgs theory, Gauss's law yields also a non-zero $A_{t}$ for most gauges. Just as in the monopole and dyon solutions, $A_{t}$ produces a back reaction on $A_{i}$ perturbatively. Although the dyon fields are time-independent, there is a net kinetic energy because $A_{t}$ is non-vanishing, so are steadily rotating (see for example the textbook of Weinberg [23]).

In our model we have rotation, i.e., a term $N^{\varphi}(t, \rho)$. If we calculate the conservation equations for the Einstein equations, one easily finds that

$$
P_{0}=\frac{1}{e} P N^{\varphi}
$$

So we obtained a kind of natural "gauge" in order to get rid of $A_{0}$ (Equation (8)). The equation for $N^{\varphi}$ decouples from the other equations.

The field equations now become

$$
\begin{aligned}
& \ddot{N}=-N^{4}\left(N^{\prime \prime}+3 \frac{N^{\prime}}{\rho}\right)+3 \frac{\dot{N}^{2}}{N}-N^{3} N^{\prime 2}+\frac{1}{\eta^{2} X^{2}+\omega^{2}}\left[3 N\left(N^{4} \omega^{\prime 2}-\dot{\omega}^{2}\right)\right. \\
&-N^{3} V-\frac{N^{5}}{\rho}\left(\omega \omega^{\prime}+\eta^{2} X X^{\prime}\right)+3 \eta^{2} N\left(N^{4} X^{\prime 2}-\dot{X}^{2}\right)+2 \frac{N}{e^{2} \rho^{2}}\left(\dot{P}^{2}-N^{4} P^{\prime 2}\right) \\
&\left.-6 \frac{N^{4}}{e^{2} \rho^{3}} N^{\prime}-\frac{1}{6} \kappa^{2} \Lambda N^{3} \omega^{4}+6 \frac{\eta^{2} P^{2} N^{3} X^{2}}{\rho^{2}}\right]+\frac{1}{\left(\eta^{2} X^{2}+\omega^{2}\right)^{2}}\left[18 \frac{\eta^{2} P^{4} X^{2} N^{3}}{e^{2} \rho^{4}}\right. \\
&\left.+3 \frac{N P^{2}}{e^{4} \rho^{4}}\left(\dot{P}^{2}-N^{4} P^{\prime 2}\right)-6 \frac{P^{2} N^{5}}{e^{2} \rho^{3}}\left(\omega \omega^{\prime}+\eta^{2} X X^{\prime}\right)\right], \\
& \ddot{\omega}= N^{4}\left(\omega^{\prime \prime}+\frac{\omega^{\prime}}{\rho}\right)+2 N^{3} \omega^{\prime} N^{\prime}+2 \frac{\dot{\omega} \dot{N}^{\prime}}{N^{2}}+\frac{\eta X N^{2}}{\omega} \frac{\mathrm{d} V}{\mathrm{~d} X} \\
&+\frac{\left[\omega\left(N^{4} \omega^{\prime 2}-\dot{\omega}^{2}\right)+\eta^{2} \omega\left(N^{4} X^{\prime 2}-\dot{X}^{2}\right)-2 \frac{\omega P^{2} N^{3}}{e^{2} \rho^{3}} N^{\prime}\right]}{\eta^{2} X^{2}+\omega^{2}} \\
&+\left.\frac{N^{2}}{3 \omega}\left(V+\frac{1}{6} \kappa^{2} \Lambda \omega^{4}\right)\left(3 \omega^{4}+5 \eta^{2} X^{2} \omega^{2}+2 \eta^{4} X^{4}\right)\right], \\
&\left(\eta^{2} X^{2}+\omega^{2}\right)^{2} \\
&\left(-2 \frac{\omega^{2} P^{2} N^{4}}{e^{2} \rho^{3}}\left(\omega \omega^{\prime}+\eta^{2} X X^{\prime}\right)+\frac{1}{2 e^{2} r^{2}} \omega\left(\dot{P}^{2}-N^{4} P^{\prime 2}\right)\right. \\
&\left.e^{2}+\eta^{2} X^{2}\right)+\frac{\eta^{2} P^{2} N^{2} X^{2} \omega}{\rho^{2}}\left(\omega^{2}+6 \frac{P^{2}}{\rho^{2}}+\eta^{2} X^{2}\right)
\end{aligned}
$$




$$
\begin{gathered}
\ddot{X}=N^{4}\left(X^{\prime \prime}+\frac{X^{\prime}}{\rho}\right)+2 N^{3} X^{\prime} N^{\prime}+2 \frac{\dot{X} \dot{N}}{N}-\frac{N^{2}}{\eta} \frac{\mathrm{d} V}{\mathrm{~d} X} \\
+\frac{\left[\eta^{2} X\left(N^{4} X^{\prime 2}-\dot{X}^{2}\right)+X\left(N^{4} \omega^{\prime 2}-\dot{\omega}^{2}\right)-2 \frac{X P^{2} N^{3}}{e^{2} \rho^{3}} N^{\prime}-\frac{X N^{2}}{3}\left(V+\frac{1}{6} \kappa^{2} \Lambda \omega^{4}\right)\right]}{\eta^{2} X^{2}+\omega^{2}} \\
+\frac{1}{\left(\eta^{2} X^{2}+\omega^{2}\right)^{2}}\left[-2 \frac{X P^{2} N^{4}}{e^{2} \rho^{3}}\left(\omega \omega^{\prime}+\eta^{2} X X^{\prime}\right)+\frac{1}{2 e^{2} \rho^{2}} X\left(\dot{P}^{2}-N^{4} P^{\prime 2}\right)\right. \\
\left.\cdot\left(\omega^{2}+2 \frac{P^{2}}{e^{2} \rho^{2}}+\eta^{2} X^{2}\right)-\frac{P^{2} N^{2} X}{\rho^{2}}\left(\omega^{4}-6 \frac{\eta^{2} X^{2} P^{2}}{e^{2} \rho^{2}}+\eta^{2} X^{2} \omega^{2}\right)\right], \\
\ddot{P}=N^{4}\left(P^{\prime \prime}-\frac{P^{\prime}}{\rho}\right)+e^{2} \eta^{2} P X^{2} N^{2}+N^{3} P^{\prime} N^{\prime}+2 \frac{\dot{P} \dot{N}}{N}-4 \frac{P N^{3} N^{\prime}}{r} \\
+2 \frac{P\left[\dot{P}^{2}-N^{4} P^{\prime 2}-2 \rho e^{2} N^{4}\left(\eta^{2} X X^{\prime}+\omega \omega^{\prime}\right)+6 \eta^{2} e^{2} N^{2} X^{2} P^{2}\right]}{\rho^{2} e^{2}\left(\eta^{2} X^{2}+\omega^{2}\right)}, \\
\left(\dot{N}^{\varphi}\right)^{\prime}=-\left(N^{\varphi}\right)^{\prime} \frac{\dot{P}}{P}, \quad\left(N^{\varphi}\right)^{\prime \prime}=-\left(N^{\varphi}\right)^{\prime}\left(\frac{P^{\prime}}{P}+\frac{1}{\rho}\right), \\
\left.N^{\prime 2}=-4 \frac{N}{\rho^{3}} N^{\prime}+2 \frac{\dot{P}^{2}}{N^{2}}-N^{2} P^{\prime 2}+6 e^{2} \eta^{2} X^{2} P^{2}-2 e^{2} \rho N^{2}\left(\eta^{2} X X^{\prime}+\omega \omega^{\prime}\right)\right]
\end{gathered}
$$

Further, we obtain from the Maxwell equations and Einstein constraint equations

$$
\dot{P}=\frac{2 \eta^{2} X \dot{X}+\omega \dot{\omega}}{\eta^{2} X^{2}+\omega^{2}-2 \frac{P^{2}}{e^{2} \rho^{2}}}, \quad P^{\prime}=\frac{2 \eta^{2} X X^{\prime}+\omega \omega^{\prime}}{\eta^{2} X^{2}+\omega^{2}-2 \frac{P^{2}}{e^{2} \rho^{2}}} .
$$

The equation for $\omega$ is obtained from the Einstein equations and the scalar equation for $\mathrm{X}$. If we substitute back the equations into the dilaton equation, we obtain the relation for the potential

$$
\frac{2}{3} V=\eta X \frac{\mathrm{d} V}{\mathrm{~d} X}+\omega \frac{\mathrm{d} V}{\mathrm{~d} \omega},
$$

From the conservation equations we then obtain

$$
\dot{V}=5 \frac{\eta^{2} X^{2} P \dot{P}}{\rho^{2}}+6 \eta \dot{X} \frac{\mathrm{d} V}{\mathrm{~d} X}+6 \dot{\omega} \frac{\mathrm{d} V}{\mathrm{~d} \omega}, \quad V^{\prime}=5 \frac{\eta^{2} X^{2} P P^{\prime}}{\rho^{2}}+6 \eta X^{\prime} \frac{\mathrm{d} V}{\mathrm{~d} X}+6 \omega^{\prime} \frac{\mathrm{d} V}{\mathrm{~d} \omega} .
$$

Sometimes, one chooses a unitary gauge in order to obtain a comparable relation (see for example Oda [24]).

\subsection{The Numerical Solution}

We can plot a numerical solution of the field equations of Section 3.1 for a set of initial and boundary values. We can choose as initial values the vacuum solution of Equation (16)-(21). This solution can easily be found exactly:

$$
\begin{aligned}
& \omega=F_{1}(\rho) \mathrm{e}^{-\frac{1}{2} k_{1} t}, \quad N=\frac{1}{F_{1}(\rho)} G(t)-\log \left(\rho^{1 / k_{1}} F_{1}(\rho)^{2 / k_{1}}\right), \\
& N^{\varphi}=H_{1}(t)+F_{2}(\rho) \mathrm{e}^{k_{1} t}, \quad F_{2}=a_{1}+a_{2} \int \frac{1}{\rho^{3} F_{1}^{2}} \mathrm{~d} \rho,
\end{aligned}
$$


where from the other PDE's a function for $F_{1}$ can be found for any $k_{1}$. We write the second order PDE system as a set of first order PDE's and used a Mathematica routine for solving the system. We checked the solution with the Cadsol-Fidisol solver. ${ }^{1}$ In Figure 2_and Figure 3 we plotted typical solutions for different initial and boundary conditions. It turns out that the solution is insensitive for the cosmological constant (as expected), but very sensitive for the value of the potential. Further, we observe that an initial wavelike function for the scalar field, induces a wavelike behavior in the dilaton field. It is not a hard task to find the initial conditions and the suitable values of the several parameters in order to obtain a regular and singular free spacetime $g_{\mu \nu}$ out of a BTZ solution $\tilde{g}_{\mu v}$ with its horizons. We already mentioned in the introduction, that the z-coordinate don't play a role in our model. So it was possible to uplift the BTZ-spacetime. We will return to this issue in connection with conformal compactification in the next sections.
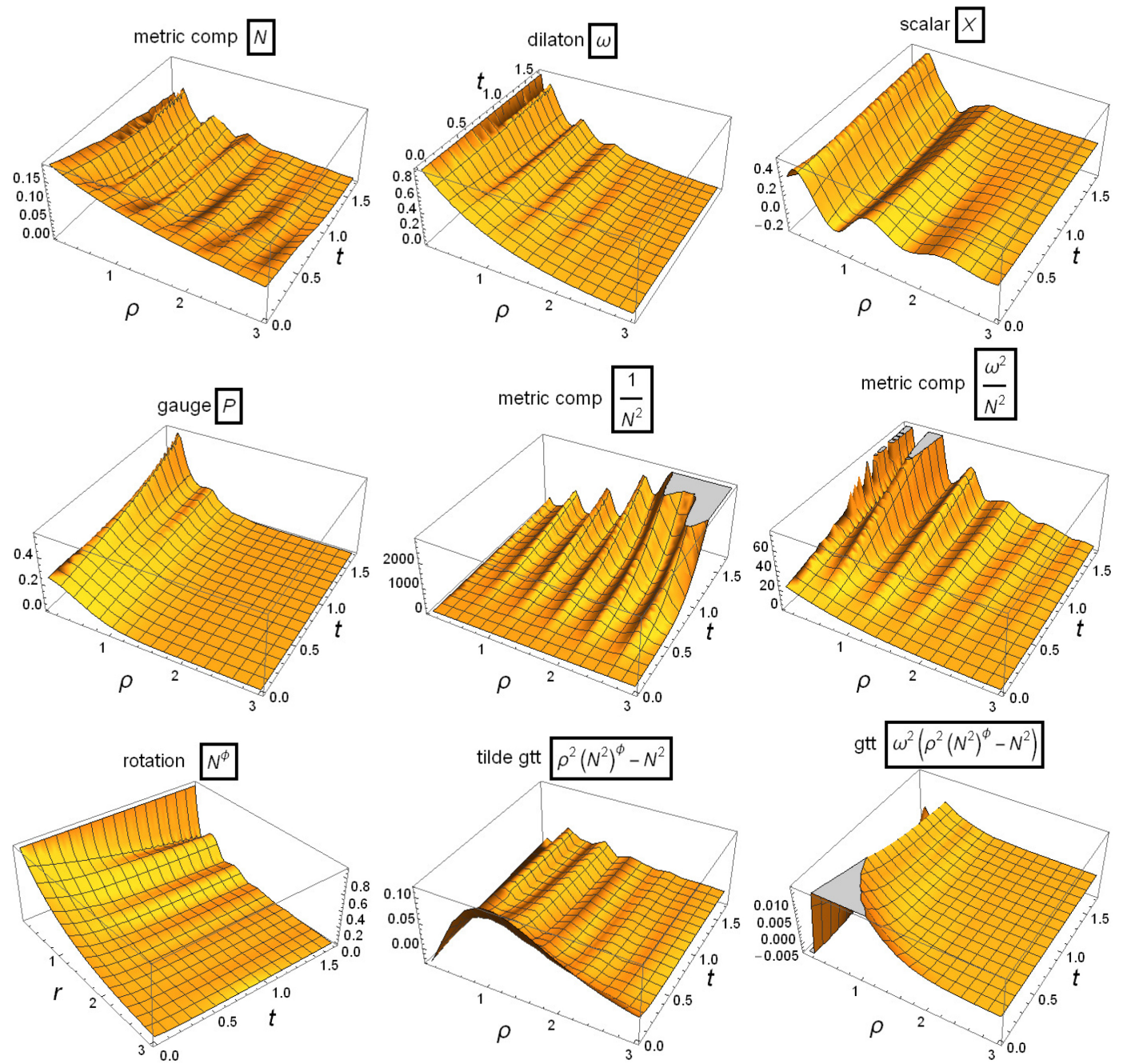

Figure 2. Example of a numerical solution of the system of Equation (16)-(21) with only for the scalar field $X$ an outgoing wavelike initial value. We used the potential from Equation (23).

${ }^{1}$ See: http://www.sai.msu.su/sal/B/2/FIDISOL.html. 

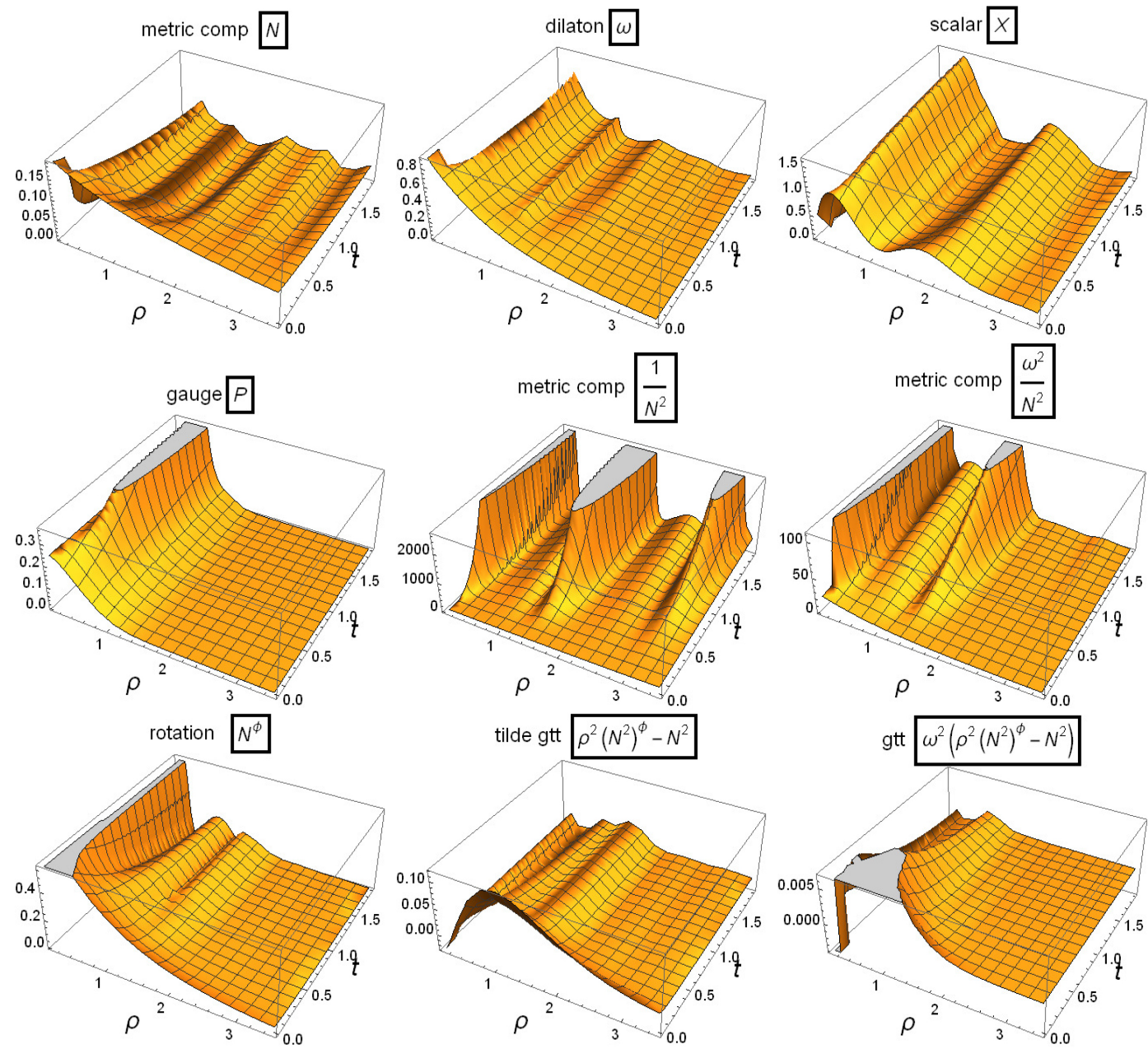

Figure 3. As Figure 2, but now with a different initial wave for the scalar field. We used the initial outgoing wave $X=\mathrm{e}^{-\rho}(\sin 4(\rho-t)+\rho)$. We observe that the wave turns quickly into a solitary wave and induces a wavelike behavior in $\omega$.

\section{An Exact Time-Dependent Vacuum BTZ Solution in Eddington-Finkelstein Coordinates in Conformal Invariant Gravity}

Quite recently, some progress was made in understanding the physics at the horizon of black holes, where quantum effects will come into play. For a mainstream treatment on this subject we refer to Parker and Toms [25].

The fundamental question is what happens with an evaporating black hole (see for example the overview article of Page [26] and references therein). It is for sure that quantum effects will resolve the distinction between the inside and outside of the black hole and the description of the hawking radiation. It will be necessary to consider the dynamical evolution of the spacetime. This can be done in a tractable way in an Eddington-Finkelstein coordinate system. One of the first attempts was the Vaidya solution [27]. In fact, the Vaidya solution is one of the non-static solutions of the Einstein field equations and is a generalization of the static Schwarzschild black hole solution. This solution is characte- 
rized by a dynamical mass function depending on the retarded time. A solution on the $(2+1)$-dimensional BTZ spacetime was recently found by Chan et al. [28] and an approximate solution by Abdolrahimi, et al. [29]. There are several ways to look at the "inside" of a black hole, or, differently formulated, how to extend maximally the Penrose diagram. Some authors use the existence of white holes, a parallel universe, or a wormhole to black-bounce transition [30] [31]. Another possibility was proposed by Susskind and Maldacena [32]. Two entangled particles (a so-called Einstein-Podolsky-Rosen or EPR pair) are connected by a wormhole (or Einstein Rosen bridge) and may be a basis for unifying general relativity and quantum mechanics. However, the two entangled black holes in regions I and II in the extended Penrose diagram, will interact via the ingoing and outgoing particles instantly. Another problem is, how to treat the connection between the observation of the in-falling observer and the outside observer, i.e., how to map the quantum states of the in- and out-going radiation in a one-to-one way. In context of conformal invariance and black hole complementarity, there is another possibility of maximal extension of the Penrose diagram as initiated by 't Hooft [33], using antipodal identification as spherical harmonics (see also 't Hooft [20] and references therein). If one doesn't want to give up locality and unitarity, one needs this approach. We can ask ourselves if some of these ideas can be applied to our spacetime. It seems possible for the Kerr spacetime [3]. However, here we are dealing with the uplifted standard $(2+1)$ BTZ spacetime. It is clear that one has to consider a dynamical evolution of the spacetime, as described in section 3.1. In the case of the BTZ black hole, the evolution of the horizons (where the inner one is the unstable Cauchy horizon) and ergo-surface outside the horizons can then be revealed.

Let us write the spacetime Equation (6) in the retarded ("outgoing") $U=t-\rho^{*}$ or advanced ("ingoing”) $V=t+\rho^{*}$ Eddington-Finkelstein coordinates

$$
\begin{aligned}
& \mathrm{d} s_{r}^{2}=\omega(U, \rho)^{2}\left[-N(U, \rho)^{2} \mathrm{~d} U^{2}-2 \mathrm{~d} U \mathrm{~d} \rho+\mathrm{d} z^{2}+\rho^{2}\left(\mathrm{~d} \xi+N^{\xi}(U, \rho) \mathrm{d} U\right)^{2}\right], \\
& \mathrm{d} s_{a}^{2}=\omega(V, \rho)^{2}\left[-N(V, \rho)^{2} \mathrm{~d} V^{2}+2 \mathrm{~d} V \mathrm{~d} \rho+\mathrm{d} z^{2}+\rho^{2}\left(\mathrm{~d} \xi+N^{\xi}(V, \rho) \mathrm{d} V\right)^{2}\right],
\end{aligned}
$$

with

$$
\begin{aligned}
& \mathrm{d} U \equiv \mathrm{d} t-\frac{\mathrm{d} \rho}{N(t, \rho)^{2}} \equiv t-\rho^{*}, \mathrm{~d} V \equiv \mathrm{d} t+\frac{\mathrm{d} \rho}{N(t, \rho)^{2}} \equiv t+\rho^{*}, \\
& \mathrm{~d} \xi \equiv \mathrm{d} \varphi-\frac{N^{\varphi}(t, \rho)}{N(t, \rho)^{2}} \mathrm{~d} \rho,
\end{aligned}
$$

with domains $U / V \in[-\infty,+\infty], \rho \in[-\infty,+\infty]$. We make no a priori assumptions for $N$ and $N^{\xi}$ (for example by writing $N=1-\frac{2 M(u)}{\sqrt{\rho^{2}+a^{2}}}$ [30]). The field equations without matter terms now reduce to (an over-dot represents $\frac{\partial}{\partial U}$ and $\left.'=\frac{\partial}{\partial \rho}\right)$ 


$$
\begin{gathered}
\omega^{\prime \prime}=\frac{2 \omega^{\prime}}{\omega}, \\
2 \omega N \omega^{\prime} N^{\prime}+3 N^{2} \omega^{\prime 2}+\frac{1}{\rho} \omega N^{2} \omega^{\prime}+2 \omega \dot{\omega}^{\prime}+2 \dot{\omega} \omega^{\prime}+\frac{1}{\rho} \omega \dot{\omega}, \\
N^{\prime \prime}=-\frac{N^{\prime 2}}{N}-\frac{4 \omega^{\prime} N^{\prime}}{\omega}-\frac{3 N \omega^{\prime 2}}{\omega^{2}}-\frac{3 N^{\prime}}{r}-\frac{3 N \omega^{\prime}}{r \omega}+4 \omega \dot{\omega}^{\prime}-\frac{2 \dot{\omega} \omega^{\prime}}{N \omega^{2}}+\frac{3 \dot{\omega}}{r N \omega}, \\
4 \dot{\omega}^{2}-2 \omega \ddot{\omega}-\frac{\omega N^{2} \dot{\omega}}{r}+\frac{\dot{N} N \omega^{2}}{r}-6 \omega^{\prime} \dot{\omega} N^{2}-2 \omega \dot{\omega} N N^{\prime}+2 \omega N \omega^{\prime} N^{\prime} \\
+3 \omega^{\prime 2}+2 \omega \omega^{\prime} N^{3} N^{\prime}+\frac{\omega \omega^{\prime} N^{4}}{r}=0, \\
\left(\dot{N}^{\xi}\right)^{\prime}=-2\left(N^{\xi}\right)^{\prime} \frac{\dot{\omega}}{\omega}, \quad\left(N^{\xi}\right)^{\prime \prime}=-2\left(N^{\xi}\right)^{\prime}\left(\frac{2 \omega^{\prime}}{\omega}+\frac{3}{r}\right),
\end{gathered}
$$

and constraint

$$
\left(N^{\xi}\right)^{\prime 2}=\frac{4}{3 \rho^{2}}\left[N N^{\prime}+N^{\prime 2}-3 N^{2} \frac{\omega^{\prime 2}}{\omega^{2}}-2 N^{2} \frac{\omega^{\prime}}{\rho \omega}+6 \frac{\omega^{\prime} \dot{\omega}}{\omega}+2 \frac{\dot{\omega}}{\rho \omega}\right]
$$

One easily finds the non-trivial solution

$$
\omega=\frac{1}{\mathrm{e}^{c_{1} U}\left(c_{2} \rho+c_{3}\right)}, \quad N^{2}= \pm c_{1} \frac{c_{3}^{2}-c_{2}^{2} \rho^{2}}{c_{2} c_{3}}, \quad N^{\xi}=F(U),
$$

with $F(U)$ an arbitrary function of $U$. This solution is consistent with the dilaton equation. Further, it is remarkable that the time dependency emerge in $\omega$ and not $N$. However, $\tilde{g}_{U U}$ depends on $U$ via $N^{\xi}$. So our metric $g_{\mu \nu}^{(4)}$ becomes (for the retarded case)

$$
\mathrm{d} s^{2}=\frac{\mathrm{e}^{-2 c_{1} U}}{\left(c_{2} \rho+c_{3}\right)^{2}}\left[ \pm \frac{c_{1}\left(c_{3}^{2}-c_{2}^{2} \rho^{2}\right)}{c_{2} c_{3}} \mathrm{~d} U^{2}-2 \mathrm{~d} U \mathrm{~d} \rho+\mathrm{d} z^{2}+\rho^{2}(\mathrm{~d} \xi+F(U) \mathrm{d} U)^{2}\right]
$$

which is flat, while $\tilde{R}^{(4)}=\frac{6 c_{1} c_{2}}{c_{3}}$. The function $F(U)$ will be fixed when matter terms are incorporated (i.e. for example, a scalar gauge field). The metric Equation (26) will then contain a term $b(U, \rho)^{2} \mathrm{~d} \varphi^{2}$ and a relation like $\left(N^{\xi}\right)^{\prime}=\frac{b}{\eta^{2} X^{2}+\omega^{2}}$ will be obtained. We can now express, for example, $U$ in $t$ and $\rho$ :

$$
U=t-\log \left[\frac{c_{2} \rho+c_{3}}{c_{2} \rho-c_{3}}\right] .
$$

So we have now a complete picture of the spacetime. We must note that this solution is rather different with respect to the vacuum Vaidya spacetime. We also are dealing here with null radiation (null matter fields or gravitational radiation) as in the case of Vaidya, but we did not make any explicit assumption for the $U$ or $V$ dependency of $\omega, N$ and $N^{\xi}$. They follow from the field equations. Further, the radiation is in the $(\rho, z)$-plane instead of the $(r, \theta)$ plane in the 
Vaidya case. It is interesting to compare our solution with the Vaidya-type solution of a spinning black hole in $(2+1)$ dimensions found by Chan in conventional GR [28]. They also find a rotation function $N^{\xi}(U)$ which is determined by an energy-momentum tensor of null spinning dust. From Equation (35), we see that the small-scale behavior (and so the dynamical apparent Cauchy horizon) is determined by

$$
\omega^{2}\left(\rho^{2} N^{\xi 2}-N^{2}\right)=\frac{\rho^{2} F(U)^{2}-\frac{c_{1}\left(c_{3}^{2}-c_{2}^{2} \rho^{2}\right)}{c_{2} c_{3}}}{\mathrm{e}^{2 c_{1} U}\left(c_{2} \rho+c_{3}\right)^{2}},
$$

and in the advanced coordinate

$$
\omega^{2}\left(\rho^{2} N^{\xi 2}-N^{2}\right)=\frac{\rho^{2} F(V)^{2}+\frac{c_{1}\left(c_{3}^{2}-c_{2}^{2} \rho^{2}\right)}{c_{2} c_{3}}}{\mathrm{e}^{2 c_{1} V}\left(c_{2} \rho+c_{3}\right)^{2}} .
$$

If we omit the dilaton factor, we obtain the expressions for $\tilde{g}_{\mu v}$. De apparent horizon is then determined (in $V$ ) by

$$
\frac{\mathrm{d} \rho}{\mathrm{d} V}=\frac{1}{2}\left(\rho^{2}\left(N^{\xi}\right)^{2}-N^{2}\right)=0,
$$

so

$$
\rho_{A H}= \pm \frac{c_{3}}{\sqrt{c_{2}\left(c_{2}-\frac{c_{3}}{c_{1}} F(V)^{2}\right)}},
$$

and in $U$

$$
\rho_{A H}= \pm \frac{c_{3}}{\sqrt{c_{2}\left(c_{2}+\frac{c_{3}}{c_{1}} F(U)^{2}\right)}} .
$$

We see that the location of apparent horizon is independent of the dilaton (so also valid for $\tilde{g}_{\mu v}$ ). However, $g_{V V}$ depends also on $\omega$, as can be seen by inspection of Equation (35), i.e., the denominator. The solution turns out to be also valid in the $(2+1)$-dimensional spacetime, i.e., $\tilde{R}^{(3)}=\frac{6 c_{1} c_{2}}{c_{3}}$ and $g_{\mu v}^{(3)}$ flat. So we can safely uplift the BTZ solution in Eddington-Finkelstein coordinates in vacuum to 4 -dimensional spacetime. We will return to this issue in the next section. In Figure 4 we plotted $\rho^{*}$ against $\rho$ and $\rho^{*}$ against $U$. The asymptote is at $\frac{c_{3}}{c_{2}}$. In Figure 5 we plotted the light cone structure. For the outward emitted signals, the slope is given by Equation (39) (in $U$ ) (for the inward, $\mathrm{d} U=0$ ). For the limiting cases, we obtain

$$
\frac{\mathrm{d} \rho}{\mathrm{d} U}=\frac{1}{2} \mathrm{e}^{-2 c_{1} U} \cdot \begin{cases}-\frac{c_{1}}{c_{2} c_{3}} & \rho \rightarrow 0 \\ \frac{c_{3} F(U)^{2}+c_{1} c_{2}}{c_{2}^{2} c_{3}} & \rho \rightarrow \infty \\ 0 & \rho=\rho_{A H}\end{cases}
$$



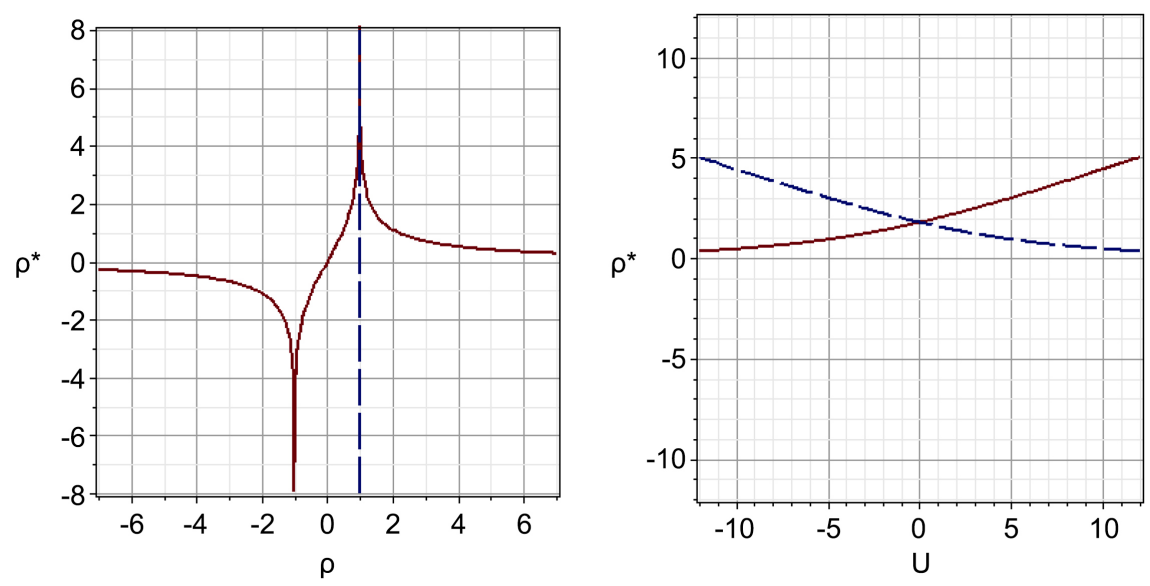

Figure 4. Left: $\rho^{*}$ as function of $\rho$. The asymptote is at $\frac{c_{3}}{c_{2}}$. Right: $\rho^{*}$ as function of $U$ for $c_{1}=c_{2}=c_{3}=1$ and $c_{4}= \pm 0.3$.
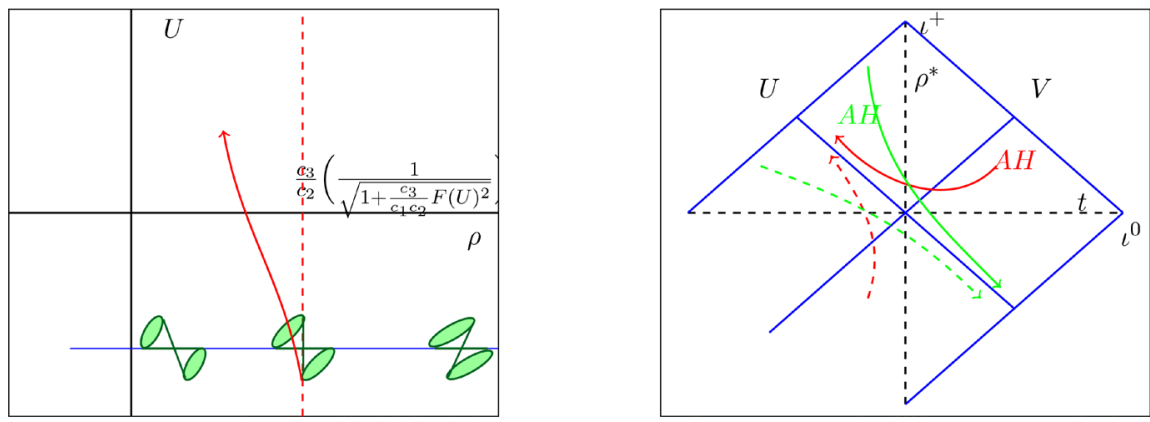

Figure 5. Left: Plot of light cone structure. The location of the apparent horizon is for small $F(U)$ at $\frac{c_{3}}{c_{2}}$. For increasing $F(U)$ it tends closer to $\rho=0$. Right: Penrose diagram for the evaporating BTZ black hole in Eddington Finkelstein coordinates $\left(U, \rho^{*}\right)$. The global location of the two pairs of the apparent horizons as function of $U$ is indicated. Note that one pair $\rho_{A H}^{*}$ enters the "future inside" region and comes from the "past inside". By the antipodal identification these regions are removed (no "inside" of the black hole) so the locations are mapped on each other. See Section 5.

For $\rho \rightarrow 0$, its value doesn't tend necessarily to $-\infty$. For increasing $U$ it could approach zero again by suitable $c_{1}$. Note that in general the location of the apparent horizon is dependent of $U$ (see Figure 5). We can express the apparent horizon also in $\rho^{*}$,

$$
\rho_{A H}^{*}=\ln \left(\frac{1+\sqrt{1+\frac{c_{3}}{c_{1} c_{2}} F(U)^{2}}}{1-\sqrt{1+\frac{c_{3}}{c_{1} c_{2}} F(U)^{2}}}\right),
$$

We can globally plot the location of the apparent horizon in a Penrose diagram, if we take for $F(U)$ for example $e^{c_{4} U}$. See Figure 5.

Let us now describe what is the meaning of the dilaton field for an infalling 
and outside observer in connection with the complementarity of the ingoing and outgoing massless particles. We will use the notion of conformal maps. The outside observed experiences a mass $\omega^{2} N^{2}$ and an evaporating black hole (in U-coordinate) by Hawking radiation (in the case of massive particles, of course, there can also be first a growing mass; we will not consider this here). This radiation is

$$
\sim \partial_{U}\left(\omega^{2} N^{2}\right)=\frac{2 c_{1}^{2}}{c_{2} c_{3}} \mathrm{e}^{-2 c_{1} U} .
$$

This blows up for $c_{1}<0$ and $U \rightarrow+\infty$. However, there is in $g_{V V}$ in the denominator the factor $\mathrm{e}^{2 c_{1} U}$. So an infalling observer crossing the apparent horizon will need a different $\omega$. The ingoing observer, passing the horizon, will NOT use the $\omega$ of the outside observer. In fact, it is locally unobservable. This happens at very small scales, when $g_{U U} \rightarrow 0$ and $\omega^{2}\left(\rho^{2} N^{\xi^{2}}-N^{2}\right) \rightarrow 0$ for $U \ll-1$ in Planck units (the ergo-surface) and there is no horizon at all (note that $\omega^{2}$ is an overall factor for $\tilde{g}_{\mu \nu}$ ). The dilaton determines the different notion of what is happening near the horizon for an infalling and outside observer. Now remember that the Ricci scalar curvature transforms under conformal transformations as $R \rightarrow \frac{1}{\Omega^{2}}\left(R-\frac{6}{\Omega} \nabla^{\alpha} \nabla_{\alpha} \Omega\right)$ and the additional freedom in $\omega$, i.e., $\omega \rightarrow \frac{1}{\Omega} \omega$. The dilaton equation of Equation (10) is an auxiliary equation in vacuum. It follows also from the Einstein equations. When matter is included, one obtains conditions on the potential (see, for example, Equation (23)). So it would be fine if we could impose $R=0$ for the local observer by using $\tilde{R}-\frac{6}{\Omega} \tilde{\nabla}^{\alpha} \tilde{\nabla}_{\alpha} \Omega=0$. One can then apply Fourier analysis of quantum mechanics and treat $\omega$ infinitesimal [34]. This is a complementarity transformation on the dilaton and switches on and off the effects these Hawking particles have on the metric.

\section{Complementarity Transformation and Conformal Compactification}

Let us first describe what is the meaning of the dilaton field for an in-falling and outside observer in connection with the complementarity of the ingoing and outgoing massless particles. We will use the notion of conformal maps. The outside observed experiences a mass $\omega^{2} N^{2}$ and an evaporating black hole (in $\mathrm{U}$-coordinate) by Hawking radiation (in the case of massive particles, of course, there can also be first a growing mass; we will not consider this here). This radiation is

$$
\sim \partial_{U}\left(\omega^{2} N^{2}\right)=\frac{2 c_{1}^{2}}{c_{2} c_{3}} \mathrm{e}^{-2 c_{1} U} .
$$

This blows up for $c_{1}<0$ and $U \rightarrow+\infty$. However, there is in $g_{V V}$ in the denominator the factor $\mathrm{e}^{2 c_{1} U}$. So an in-falling observer crossing the apparent ho- 
rizon will need a different $\omega$. The ingoing observer, passing the horizon, will NOT use the $\omega$ of the outside observer. In fact, it is locally unobservable. This happens at very small scales, when $g_{U U} \rightarrow 0$ and $\omega^{2}\left(\rho^{2} N^{\xi 2}-N^{2}\right) \rightarrow 0$ for $U \ll-1$ in Planck units (the ergo-surface) and there is no horizon at all (note that $\omega^{2}$ is an overall factor for $\tilde{g}_{\mu v}$ ). The dilaton determines the different notion of what is happening near the horizon for an in-falling and outside observer. Now remember that the Ricci scalar curvature transforms under conformal transformations as $R \rightarrow \frac{1}{\Omega^{2}}\left(R-\frac{6}{\Omega} \nabla^{\alpha} \nabla_{\alpha} \Omega\right)$ and the additional freedom in $\omega$, i.e., $\omega \rightarrow \frac{1}{\Omega} \omega$. The dilaton equation of Equation (10) is an auxiliary equation in vacuum. It follows also from the Einstein equations. When matter is included, one obtains conditions on the potential (see, for example, Equation (23)). So it would be fine if we could impose $R=0$ for the local observer by using $\tilde{R}-\frac{6}{\Omega} \tilde{\nabla}^{\alpha} \tilde{\nabla}_{\alpha} \Omega=0$. One can then apply Fourier analysis of quantum mechanics and treat $\omega$ infinitesimal [34]. This is a complementarity transformation on the dilaton and switches on and off the effects these Hawking particles have on the metric.

Let us return to the conformal mapping in more detail. We know that in Minkowski spacetime the conformal map preserves the light-cone structure and so the null geodesics (i.e., the affine parameter). The conformal group in Minkowski, however, does not act as linear transformations, so one needs a trick (see, for example Felsager [21], section 10.3).

One starts with a pseudo-Cartesian space $R^{1} \otimes R^{1}$ (for example our $(x, z)$ ). One then enlarge first the pseudo-Cartesian space by adding a "null"-cone at infinity. So one compactifies the plane in $R^{2}$. In order to apply the conformal transformation of inversion, one considers the unit sphere $S^{1}$ and map $R^{1}$ onto $S^{1}-\{N\}$. If we want to apply all the conformal transformations, then we must enlarge the pseudo-Cartesian space by adding two extra dimensions $(t, y)$, (later, we replace $\mathrm{x}$ by $x=\rho \sin \varphi$ and $y=\rho \cos \varphi$, to get back our axially symmetric spacetime coordinates $(t, \rho, z, \varphi))$. The goal is then to embed the pseudo-Cartesian space $R^{1} \otimes R^{1}$ as a subset of $R^{2} \otimes R^{2}$. We define $M$ as the intersection of the null cone $K$ (in $R^{2} \otimes R^{2}$ ) with the hyperplane $\rho-t=1$ (or $\rho+t$ ) and define an isometry $F: R^{1} \otimes R^{1} \rightarrow M$. Further, one works in the particular section of $M, M\left(R^{1} \otimes R^{1}\right)$. Because $F$ induces a coordinate system on $M$, one can construct characteristic lines. There are characteristic lines that are parallel to $\rho-t=1$ and are generated by null vectors where $\rho=t$. There is a one-to-one correspondence between these lines missing $M\left(R^{1} \otimes R^{1}\right)$ and points on $K$ in $R^{1} \otimes R^{1}$. So they represent points on the null cone at infinity. One can proof [21] that local sections $N_{1}$ and $N_{2}$ on the null cone which intersect characteristic lines at most once, can be mapped onto each other by a conformal map obtained by projection along the characteristic lines. If we would now try to project $M\left(R^{1} \otimes R^{1}\right)$ onto a suitable subsection of $K$, then it turns out that it is 
not possible to find a single section that is intersected exactly once by each characteristic line. Instead one can consider $N$ as the product of two unit spheres in $M\left(R^{2} \otimes R^{2}\right)$, i.e., $N$ becomes a hyper-torus $S^{1} \otimes S^{1}$ and each characteristic line will then intersect $K$ twice in antipodal points. So each point in $M\left(R^{1} \otimes R^{1}\right)$ is represented by a pair of antipodal points on $S^{1} \otimes S^{1}$. The projection is a conformal map. The procedure here described is called a conformal compactification of $M\left(R^{1} \otimes R^{1}\right)$. In our case the antipodal identification is $(U, V, z, \varphi) \rightarrow(-U,-V,-z, \varphi+\pi)$. The points are not physically distinct events, but identical and are different representations of one black hole. In fact, there is no inside of the black hole. The price is that the manifold is not time-orientable for $\rho<\rho_{A H}$. When the evaporation process speeds up, we observe from Equation (40) that the two horizons approach zero for increasing $F(U)$, which is assumable. Moreover

$$
\lim _{\rho \rightarrow 0} g_{U U} \rightarrow \pm \frac{c_{1}}{c_{2} c_{3} \mathrm{e}^{2 c_{1} U}}
$$

where in the denominator appears the exponential factor from the dilaton. So $\omega$ determines the scale as function of $U$ the local observer experiences. Note that on "the other side" (in the Penrose picture region II), $U$ change sign and the righthand side of Equation (46) becomes $\frac{c_{1}}{c_{2} c_{3} \mathrm{e}^{-2 c_{1} U}}$.

We found in section 4 that the solution of the BTZ spacetime in $4 \mathrm{D}$ in Eddington-Finkelstein coordinates in conformally invariant gravity is identical to the $3 \mathrm{D}$ case, where we omitted the $d z^{2}$. That is curious, because we can still apply the conformal compactification (conformal transformations) and the antipodal identifications in 4D spacetime sketched above. Further, we obtained a flat $g_{\mu v}$ out of the "un-physical" $\tilde{g}_{\mu v}$, which resembles the original BTZ-black hole (without the need of a cosmological constant).

Some notes can be made about the connection with the gravitational back-reaction. In the non-vacuum situation of section 3.2, the back-reaction is quite clear. In the vacuum case, there will be a shift in the location of the apparent horizon after the emission of null radiation (Equation (45)). This can be made clear in the Penrose diagram, as was also found in the time-dependent Vaidya spacetime in connection with black-bounces and traversable wormholes [30] [31]. In the conformally invariant model and the antipodal approach, however, one doesn't need such extreme escape. This shift will be related to the $c_{i}$ in (Equation (45)), just as the scalar curvature of $\tilde{g}_{\mu v}$ was related to $c_{i}$, i.e., $\tilde{R}^{(4)}=\frac{6 c_{1} c_{2}}{c_{3}}$. A comparable effect was found in the counterpart model of the cylindrical radiating Lewis-van Stockum solution (in $(\rho, t)$ coordinates) and Einstein-Rosen pulse-wave solution [35]. This solution is obtained from the stationary $(z, \rho)$ spacetime where one replaces $t \rightarrow i z, z \rightarrow i t$ and $J \rightarrow i J$. This solution has, however, reflection symmetry, $\varphi \rightarrow-\varphi, z \rightarrow-z$. A curious feature of the solution is the fact that the Kretschmann scalar becomes zero for two dif- 
ferent values of a constant in the exact solution. In some sense, the spacetime returns to his original status after the emission of the pulse wave. The relation with the antipodal symmetry is current under investigation by the author.

\section{Conclusions}

Using conformally invariant gravity, a new solution is found for the uplifted BTZ spacetime, without a cosmological constant. The solution shows some different features with respect to the standard BTZ solution. In the non-vacuum situation, where a scalar-gauge field is present, a numerical solution is presented on a spacetime where one writes the metric as $g_{\mu v}=\omega^{2} \tilde{g}_{\mu \nu}$, with $\omega$ a dilaton field, to be treated on equal footing with the scalar field and $\tilde{g}_{\mu v}$ an "un-physical" spacetime. The effect of $\omega$ on the behavior of the solution is evident. An outgoing wave-like initial value for the scalar field induces a wave-like response in the dilaton field and pushes the apparent horizon closer to $\rho=0$. The solution depends critically on the shape of the potential. The solution can be used to investigate what happens with the spacetime of an evaporating black hole through Hawking radiation. In the vacuum situation in Eddington-Finkelstein coordinates, an exact solution is found for the $(2+1)$-dimensional case as well as for the uplifted situation. The "un-physical" $\tilde{g}_{\mu v}$ (BTZ) solution has a non-zero Ricci scalar, while $g_{\mu v}$ is flat.

There is possible a link with the antipodal identification. Antipodal mapping is inevitable if one wants to maintain unitarity during quantum mechanical calculations on the Hawking particles. The antipodal identification can be represented as a conformal transformation generated from the pseudo-orthogonal matrices of $O(3,3)$, i.e., the conformal group. Each conformal transformation in this group can be presented by a pair of antipodal matrices. This was the main reason to investigate in this manuscript the dynamics of the BTZ black hole in conformally invariant gravity. In the conformally invariant approach, the dilaton field plays a fundamental role. We find that as soon its value is fixed (by the global spacetime after choosing the coordinate frame), the local observer experiences scales. Moreover, we find that it also plays a role in the antipodal mapping. If we substitute the apparent horizon $\rho_{A H}$ (Equation (41)) into $\omega$ (Equation (34)) at the horizon, we can then compare $\omega$ on both sides of the horizon by replacing $U$ by $-U$. By imposing proper matching conditions, one could obtain restrictions on $F(U)$.

We do not pretend that our model is a new description of the physics of an evaporating BTZ black hole. We have tried to compare conformally invariant gravity solutions of the $(2+1)$-dimensional BTZ black hole solution and its uplifted counterpart model with the results of former results on black hole studies. Especially the antipodal identification seems to fit well in our model.

\section{Conflicts of Interest}

The author declares no conflicts of interest regarding the publication of this paper. 


\section{References}

[1] Baňados, M., Henneaux, M., Teitelboim, C. and Zanelli, J. (1993) Physical Review $D$, 50, 1506. https://doi.org/10.1103/PhysRevD.48.1506

[2] Carlib, S. (1995) Classical and Quantum Gravity, 12, 2853. https://doi.org/10.1088/0264-9381/12/12/005

[3] Compère, G. (2019) Advanced Lectures on General Relativity (Lecture Notes in Physics 952). Springer, Heidelberg. https://doi.org/10.1007/978-3-030-04260-8

[4] Deser, S., Jackiw, R. and 't Hooft, G. (1992) Physical Review Letters, 68, 267. https://doi.org/10.1103/PhysRevLett.68.267

[5] Garfinkle, D. (1985) Physical Review D, 32, 1323. https://doi.org/10.1103/PhysRevD.32.1323

[6] Maldacena, J. (1999) Advances in Theoretical and Mathematical Physics, 2, 231. https://doi.org/10.1088/1126-6708/1999/02/011

[7] Strominger, A. (1997) JHEP, 1998, 009. https://doi.org/10.1088/1126-6708/1998/02/009

[8] Slagter, R.J. and Larosh, J. (2019) On the BTZ Black Hole and the Spinning Cosmic String. arXiv: gr-qc/191206222

[9] Slagter, R.J. and Duston, C.L. (2020) International Journal of Modern Physics A, 35, Article ID: 2050024. https://doi.org/10.1142/S0217751X20500244

[10] Weyl, H. (1918) Mathematische Zeitschrift, 2, 384. https://doi.org/10.1007/BF01199420

[11] Wald, R.M. (2009) General Relativity. Univ. Press, Chicago.

[12] 't Hooft, G. (2010) The Conformal Constraint in Canonical Quantum Gravity. arXiv:gr-qc/10110061

[13] 't Hooft, G. (2011) Foundations of Physics, 41, 1829. https://doi.org/10.1007/s10701-011-9586-8

[14] Bars, I., Steinhardt, P. and Turok, N. (2014) Physical Review D, 89, Article ID: 043515. https://doi.org/10.1103/PhysRevD.89.043515

[15] Mannheim, P.D. (2005) Alternatives to Dark Matter and Dark Energy.

[16] Slagter, R.J. and Pan, S. (2016) Foundations of Physics, 46, 1075-1089. https://doi.org/10.1007/s10701-016-0002-2

[17] 't Hooft, G. and Veltman, M. (1974) Annales de P Institut Henri Poincaré, 20, 69.

[18] Stelle, K.S. (1977) Physical Review D, 16, 953. https://doi.org/10.1103/PhysRevD.16.953

[19] Duff, M.J. (1993) Twenty Years of the Weyl Anomaly.

[20] 't Hooft, G. (2019) The Quantum Black Hole as a Theoretical Lab, a Pedagogical Treatment of a New Approach.

[21] Felsager, B. (1998) Geometry, Particles and Fields. Odense Univ. Press, Odense. https://doi.org/10.1007/978-1-4612-0631-6

[22] 't Hooft, G. (2015) Local Conformal Symmetry: the Missing Symmetry Component for Space and Time. arXiv: gr-qc/14106675v1.

[23] Weinberg, E.J. (2012) Classical Solutions in Quantum Field Theory. Cambridge University Press, Cambridge.

[24] Oda, I. (2015) Conformal Higgs Gravity. arXiv: gr-cq/150506760

[25] Parker, L.E. and Toms, D.J. (2009) Quantum Field Theory in Curved Spacetime. 
Cambridge University Press, Cambridge. https://doi.org/10.1017/CBO9780511813924

[26] Page, D.N. (2004) New Journal of Physics, 7, 203. https://doi.org/10.1088/1367-2630/7/1/203

[27] Vaidya, P.C. (1943) Current Science, 12, 183. https://doi.org/10.1525/as.1943.12.18.01p1268z

[28] Chan, J.S.F., Chan, K.C.H. and Mann, R.B. (1996) Physical Review D, 54, 1535. https://doi.org/10.1103/PhysRevD.54.1535

[29] Abdolrahimi, S., Page, D.N. and Tzounis, C. (2019) Ingoing Eddington-Finkelstein Metric of an Evaporating Black Hole. arXiv: hep-th/160705280V4

[30] Simpson, A., Martin-Moruna, P. and Visser, M. (2019) Classical and Quantum Gravity, 36, Article ID: 145007. https://doi.org/10.1088/1361-6382/ab28a5

[31] Simpson, A. and Visser, M. (2019) Black-Bounce to Traversable Wormhole. arXiv: gr-qc/181207114v3

[32] Maldacena, J. and Susskind, L. (2013) Fortschritte der Physik, 61, 781. https://doi.org/10.1002/prop.201300020

[33] 't Hooft, G. (2016) Foundations of Physics, 46, 1185. https://doi.org/10.1007/s10701-016-0014-y

[34] 't Hooft, G. (2009) Quantum Gravity without Space-Time Singularities or Horizons. arXiv: gr-qc/09093426

[35] Islam, J.N. (1985) Rotating Fields in General Relativity. Cambridge University Press, Cambridge. https://doi.org/10.1017/CBO9780511735738 